\title{
Addressing the Fuel Resistance of Hot Mix Asphalt by an Enhanced Test Method
}

\author{
Bernhard Hofko and Ronald Blab \\ Institute of Transportation, Vienna University of Technology, Vienna 1040, Austria
}

\begin{abstract}
In areas where HMA (hot mix asphalt) is likely to be exposed by any form of mineral oil, the layer has to withstand the attack of these substances in order not to damage the construction. The European standard EN 12697-43 provides a test procedure to determine the resistance of HMA to fuel. The paper reviews this method thoroughly. A completely revised and simplified test device for the brush test was developed meeting the requirements of the standard and creating results with a high repeatability at the same time. The test conditions given by the standard such as the exposure to fuel, cleaning of the specimen after exposure or the contact pressure of the brush were varied to isolate those test conditions with a substantial influence on the result. The research revealed that in the standard some conditions with a rather small influence are set quite strictly while other conditions with a distinct influence on the result are not defined with the required accuracy to obtain comparable and repeatable results. The paper presents suggestions for the improvement of the test method and the standard itself in respect to the layout of the test device and the definition of important test conditions to enhance the outcome of the EN 12697-43.
\end{abstract}

Key words: Fuel resistance, hot mix asphalt, abrasion test.

\section{Introduction}

HMA (hot mix asphalt) is mainly used in road construction for flexible pavements but is also suited for traffic areas on airports, parking lots, or at gas stations. When these layers are subjected to any form of mineral oil, such as kerosene, diesel or benzine (gasoline), the HMA has to resist fuel exposure in order not to damage the construction.

For the assessment of the resistance to fuel, different test methods have been developed in the last 20 years [1-3]. The tests can be divided into two main groups or a combination of both. Chemical methods include tests where asphalt or bitumen specimens are directly exposed to fuel without any mechanical loading. The effect of the fuel exposure is quantified by different means, e.g. the penetration depth of the liquid, the mass loss of asphalt specimens or the chemical change of the bitumen itself.

In many cases, these chemical methods are

Corresponding author: Bernhard Hofko, Dr., research fields: material science, asphalt mixes, bitumen and rheology. E-mail: bernhard.hofko@tuwien.ac.at. combined with subsequent mechanical testing. These tests include the determination of strength or stiffness parameters and the mass loss due to abrasive loading. In some cases, reference specimens not exposed to fuel are also tested to isolate the influence of the fuel. From the wide variety of test methods developed in the last decades, the European standard EN 12697-43 (2005) "resistance to fuel" chose a direct exposure of asphalt specimens to fuel followed by a brush test to obtain mass loss due to chemical and abrasive loading. The brush test is often used in research to determine resistance of HMA to fuel [4]. It can be seen as a modification of the wet track abrasion test [5].

In an extensive research program, the contents of EN 12697-43 (2005) were critically reviewed and test conditions systemically varied to isolate those conditions with a distinct influence on the test results. The findings of the project can account for an efficient improvement of the standard.

\section{Resistance to Fuel acc. to EN 12697-43}

According to EN 12697-43 (2005), a cylindrical 
specimen with a known mass is immersed partly in a bath with the specified fuel. For bituminous mixtures with paving grade bitumen, the specimens are exposed for 24 hours, whereas for specimens with polymer modified bitumen the exposure should last for 72 hours. The standard states that the immersion time for mixes with paving grade bitumen and polymer modified bitumen should be $24 \mathrm{~h}$ if the performance of mixes with both bitumen types is compared. For each material, at least three specimens are to be tested. In case of porous asphalt, the specimens shall have a diameter of $(150 \pm 2) \mathrm{mm}$, for other asphalt mixes the diameter is set to $(100 \pm 2) \mathrm{mm}$. According to EN 12697-43 (2005), the height can range between 40 $\mathrm{mm}$ and $60 \mathrm{~mm}$.

Before testing the dry mass of the specimen is recorded as $\mathrm{m}_{1}$. After the exposure, specimens are cleaned with water and dried for another 24 hours at $25{ }^{\circ} \mathrm{C}$. After drying the mass of the specimen is measured as $\mathrm{m}_{2}$ and the immersed surface is inspected by visual means. The immersed surface is then subjected to abrasion by a steel brush, which is moving in epicycloid passages over the surface. After $30 \mathrm{~s}\left(\mathrm{~m}_{3}\right), 60 \mathrm{~s}\left(\mathrm{~m}_{4}\right)$ and $120 \mathrm{~s}\left(\mathrm{~m}_{5}\right)$, the remaining mass of the specimen is recorded. The material loss after the immersion and the brush test are used as parameters to quantify the resistance to the particular fuel. Therefore, two parameters are introduced in EN 12697-43 (2005):

$$
A=\frac{m_{1}-m_{2}}{m_{1}} \cdot 100[\%]
$$

$$
B=\frac{m_{2}-m_{5}}{m_{2}} \cdot 100[\%]
$$

where, parameter $A$ determines the mass loss due to exposure to fuel (chemical loading), whereas parameter $B$ quantifies the mass loss due to abrasive loading. The resistance is characterized by a combination of the two parameters in EN 12697-43 (2005):

$$
\begin{aligned}
& A \leq 5 \% \text { and } B<1 \% \text { : good resistance; } \\
& A \leq 5 \% \text { and } 1 \% \leq B \leq 5 \% \text { : moderate resistance; } \\
& A>5 \% \text { or } B>5 \% \text { : poor resistance. }
\end{aligned}
$$

\section{Materials and Test Program}

Within the research program, two different mix types were tested. One was an AC $11 \mathrm{PmB}$ 45/80-65 asphalt concrete with a maximum aggregate size of 11 $\mathrm{mm}$ and a polymer modified binder PmB 45/80-65 acc. to ONORM B 3613. The other mix was an AC 11 PmB 45/80 FR (fuel resistance). The basic bitumen is a PmB 45/80-65 plus additives to increase the FR. The binder content was varied from $5.0 \%$ to $5.6 \%(\mathrm{~m} / \mathrm{m})$. The target volume of air voids ranged from $3.0 \%$ to $7.0 \%(\mathrm{v} / \mathrm{v})$. An overview is given by Table 1 .

Table 2 shows the variation of test parameter within the project. Specimens were prepared from Marshall compaction (EN 12697-30) as well as from slabs compacted by roller compaction (EN 12697-33). Three different methods of specimen cleaning after exposure (W1 to W3) were investigated (see Section 4.3). Furthermore, the static loading for the abrasion test was varied and three different types of fuel were

\begin{tabular}{|c|c|c|c|c|}
\hline Specimen preparation & Method of specimen cleaning & Duration of exposure $(\mathrm{h})$ & static loading $(\mathrm{N})$ & Fuel type \\
\hline \multirow{2}{*}{ Marshall compaction } & W1 & \multirow{2}{*}{24} & \multirow{2}{*}{140} & Kerosene \\
\hline & W2 & & & Diesel \\
\hline Roller compaction & W3 & 72 & 210 & Benzine \\
\hline
\end{tabular}
analyzed.

Table 1 Materials used within the research.

\begin{tabular}{lll}
\hline Mix type & Binder content $(\%(\mathrm{~m} / \mathrm{m}))$ & Volume of air voids $(\%(\mathrm{v} / \mathrm{v}))$ \\
\hline AC 11 PmB 45/80-65 & 5.0 & 3.0 \\
AC 11 PmB 45/80 FR & 5.3 & 5.0 \\
\hline
\end{tabular}

Table 2 Test parameters. 


\section{Review of EN 12697-43}

Reviewing the test procedure given by the standard, this section provides detailed suggestions for improvements:

- Preparation of specimens;

- Dimensions of specimens in relation to fuel exposure;

- Cleaning of the specimens after exposure;

- Determination of fuel resistance acc. to new parameter $C$;

- The testing device for the brush test.

\subsection{Preparation of Specimens}

Acc. to EN 12697-43, the mix can be compacted by impact (Marshall) or gyratory compactor (EN 12697-30 and 12697-31). Also cored specimens from road pavements can be used. Roller compaction (EN 12697-33) is not a given option by the standard. A comparison within the research project showed that the difference in results between Marshall specimens and specimens cored from HMA-slabs compacted by roller compaction is not significant. As an example the left diagram in Fig. 1 shows the mass loss after exposure (parameter $A$ ) of four specimens for each preparation method vs. the volume of air voids. Thus, it is suggested that all three compaction methods are implemented in EN 12697-43, especially because the roller compactor reproduces the compaction used in road construction in the most reliable way [6].

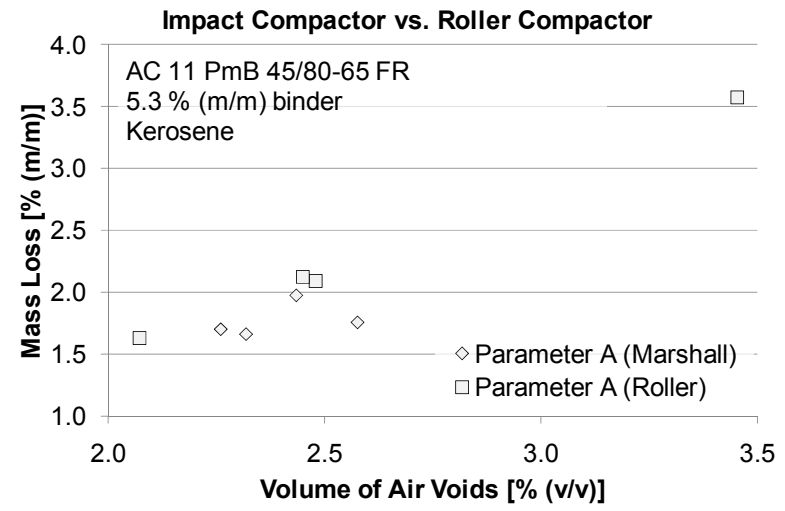

4.2 Dimensions of Specimens in Relation with Fuel Exposure

EN 12697-43 demands to place each specimen in a beaker glass in an amount of fuel so that the specimen is immersed $35 \mathrm{~mm}$ in the fuel. As the height of the specimen can range between $40 \mathrm{~mm}$ and $60 \mathrm{~mm}$ but the immersion of the specimen is fixed to $35 \mathrm{~mm}$ of fuel, the ratio of specimen volume vs. immersed volume ranges from $58 \%$ to $88 \%$. This means that smaller specimens are exposed to a much higher extent to fuel leading to higher mass losses. Thus, results from specimens with different heights cannot be compared.

The recommendation is to set the height of the specimen in the standard more strictly (e.g., $63.5 \pm 2$ $\mathrm{mm}$ ) or set the immersed part of the specimens relative to the specimen's height (e.g., $50 \% \pm 1 \%$ ).

\subsection{Cleaning of Specimens after Exposure}

After exposure, the specimens have to be cleaned from the fuel. The standard demands to "wash the specimen with water until the acidity of water $\mathrm{pH}$ is $(7.0 \pm 0.5)$ ". This wording is imprecise. After exposure, part of the binder has been dissolved by the fuel, so the surface of the immersed part of the specimen shows more or less loose stones. Depending on the method of washing, more or less of these stones are washed away leading to different results for parameter $A$ for the same material. To demonstrate

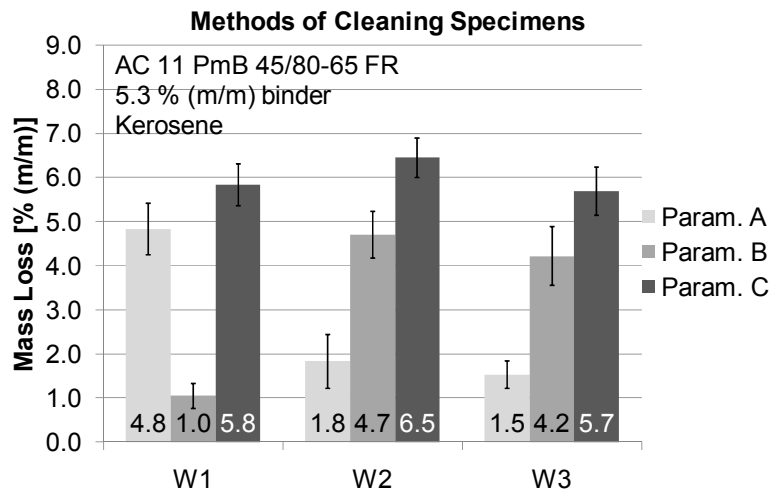

Fig. 1 Results of specimens from impactor and roller compactor (left); impact of cleaning method on results (right). 
this, within the research project three different methods of washing were investigated. One method was to clean the specimens under flowing water and removing loose aggregates "mechanically" by hand (W1, Fig. 2a). Another way of washing included cleaning the specimen under flowing water but removing loose aggregates only carefully with a hair pencil (W2, Fig. 2b). The third way was to clean the specimen just by putting it into a small box with water not directly under jet of water until the required acidity of water was reached without any "mechanical" removing of loose aggregates (W3, Fig. 2c).

The impact of the method of cleaning-especially removing of loose particles - on the results is crucial. Results for the three different methods are shown in the right diagram in Fig. 2. For each method, four specimens were tested. As the volume of air voids of all specimens is similar ( $M V: 2.5 \%(\mathrm{v} / \mathrm{v}), S D: 0.2 \%$ (v/v)), the diagram shows the $M V$ (mean values) and $S D$ (standard deviations) for each method and the parameters $A$ and $B$.

It is obvious that the most aggressive way of cleaning (W1) leads to the highest mass loss after exposure (parameter $A$ ). Methods W2 and W3 lead to the similar results. Regarding the mass loss after brush testing, the situation is reversed. The specimens cleaned aggressively now show low losses, whereas specimens cleaned according to $\mathrm{W} 2$ and $\mathrm{W} 3$ lead to higher mass losses (parameter $B$ ). If the parameters $A$ and $B$ are combined to an effective mass loss after exposure and brush test (indicated as parameter $C$ in the right diagram in Fig.1), comparable results are received independent of the method of washing. The scattering of the results in terms of $S D$ is not dependent on the method of washing.

It is strongly recommended that the method of cleaning after exposure is precisely given by the standard in the future. As the parameter $A$ should only quantify the mass loss of a material when exposed to a fuel substance without any abrasive loading, the specimens should be cleaned carefully either by washing it under flowing water without removing loose aggregates by hand or by putting it into a box keeping it out of the direct jet of water. The standard should state clearly that the surface of the specimen should not be rubbed while cleaning with water and additional it must be explained how to remove loose particles. Furthermore, we recommend altering the determination of the fuel resistance. Today, EN 12697-43 obtains the fuel resistance by parameters $A$ and $B$. As shown, different methods of washing lead to a wide range of these parameters for the same material. Thus, the results cannot be seen as comparable or repeatable. It is recommended to implement a new parameter $C$ as presented in the next section.

\subsection{Determination of Fuel Resistance acc. to New} Parameter $C$

The influence of the method of cleaning the specimens after exposure on the fuel resistance can be overcome by introducing a parameter $C$ :

$$
C=\frac{m_{1}-m_{5}}{m_{1}}[\%]
$$

Parameter $C$ indicates the total loss of mass after

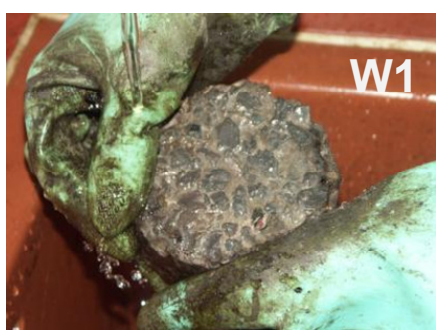

(a)

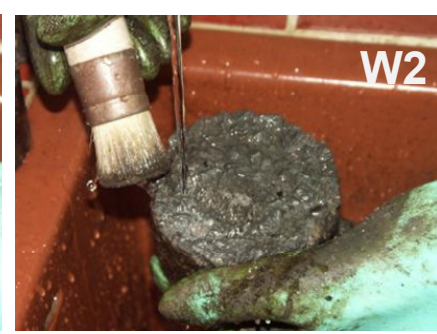

(b)

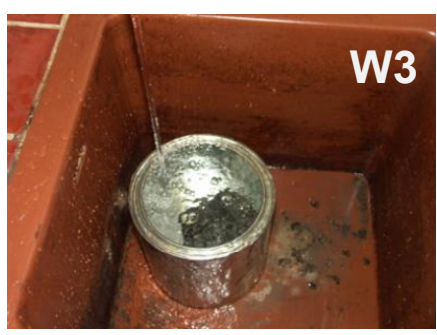

(c)

Fig. 2 Three methods of cleaning specimens after exposure. 
chemical and abrasive loading. As shown in the above section, this parameter is independent of the method of cleaning. The resistance can be characterized as follows:

$C<6 \%$ : good resistance;

$6 \% \leq C \leq 10 \%$ : moderate resistance;

$C>10 \%$ : poor resistance.

Parameters $A$ and $B$ will still be obtained as an information how the tested material reacts to fuel exposure and abrasive loading, respectively.

\subsection{Testing Device for Brush Test}

For the brush test, EN 12697-43 provides an example device including a Hobart mixer which should be able to carry out epicyclical motion covering an area of $100 \mathrm{~mm}$ in diameter with a rotation speed of $60 \pm 2 \mathrm{rpm}$. The mixer in this case carries the brush. To fix the specimen under the brush and to realize a constant pressure of the specimen to the brush during the test, a frame is presented in the standard working with compressed air in Fig. 3. On closer examination, this system cannot guarantee that the pressure produced by the compressed air is fully effective for the contact pressure of the specimen to the brush. The skin friction of the steel rings and the wedges used to fix the specimen within the frame will transfer part of the forces produced by the compressor. Furthermore, the contact pressure cannot be kept constant throughout the test when the height of the specimen is reduced due to abrasion of the brush since the metal ring holding the specimen is fixed in vertical direction by a thread bar and nut before the brushing starts.

As a result of this analysis, a completely revised device for the brush test was constructed. An overview of the new brush testing device is depicted in Fig. 4. A standard milling machine was adapted to meet the requirements of the standard. The hand wheel usually used to set the milling head was replaced by a deflection pulley $(\mathrm{G})$ carrying weights (F) that apply the contact pressure from above to the specimen using gravity. The milling head itself was replaced by a clamp for the brush. One main advantage of the system is that the brush can be moved in vertical direction throughout the test to ensure a constant contact pressure. The change in the height of the specimen due to abrasion is now adjusted by the moving brush. The eccentricity of the epicyclical motion of the brush can also be set in a wide range, so that specimens with a diameter of 100 $\mathrm{mm}$ and $150 \mathrm{~mm}$ can be brushed covering the entire surface. The specimen itself is fixed by clamping jaws (D) with a variable diameter. Thus, the position of the specimen is fixed and always centered below the brush.
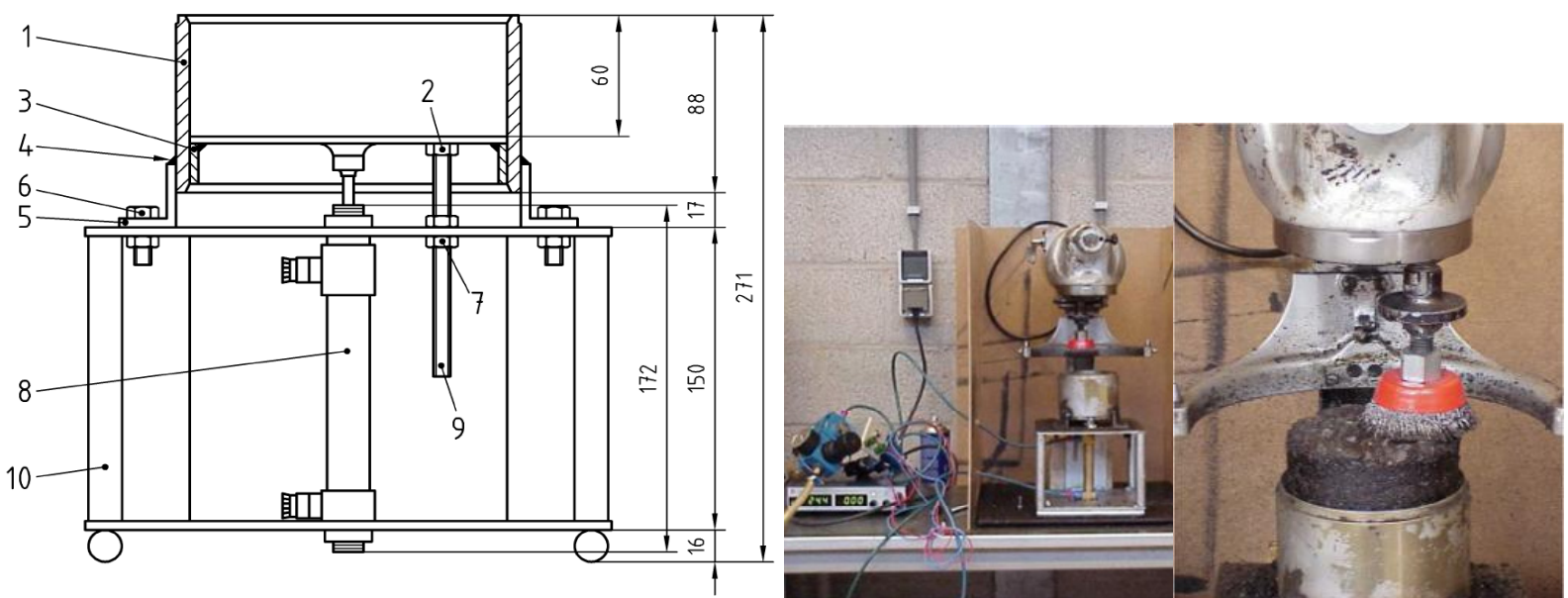

Fig. 3 Example for the frame for brush testing (EN-12697-43) (left); brush test device acc. to EN 12697-43 and detail of the brush test [7] (right). 


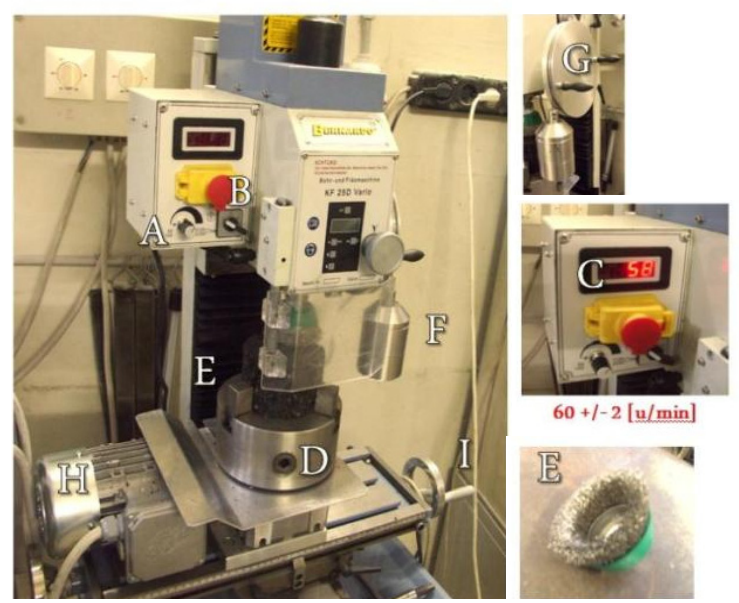

A digital speed control

B emergency shutdown

C speed display

D clamping jaws

E brush acc. to EN 1269743

F weight at deflection pulley

G deflection pulley

$\mathrm{H}$ engine for clamping jaws

I hand wheel to position the clamping jaws for epicyclical motion.

Fig. 4 Improved brush test device from a remodeled milling machine.

As the drawbacks of the device given by the standard are overcome by the test machine presented above, tests to assess the resistance of fuel of HMA could now be carried out producing comparable and repeatable results with an easy-to-handle device that guarantees to meet all requirements given by the EN 12697-43.

\section{Further Influences on the Test Results}

In the first test program using the new device, the main influences of test conditions set by the standard were isolated. Besides different methods of specimen preparation and cleaning of the specimens after exposure already analyzed above, the influence of the duration of exposure, different contact pressures for the brush test and different fuel types were investigated.

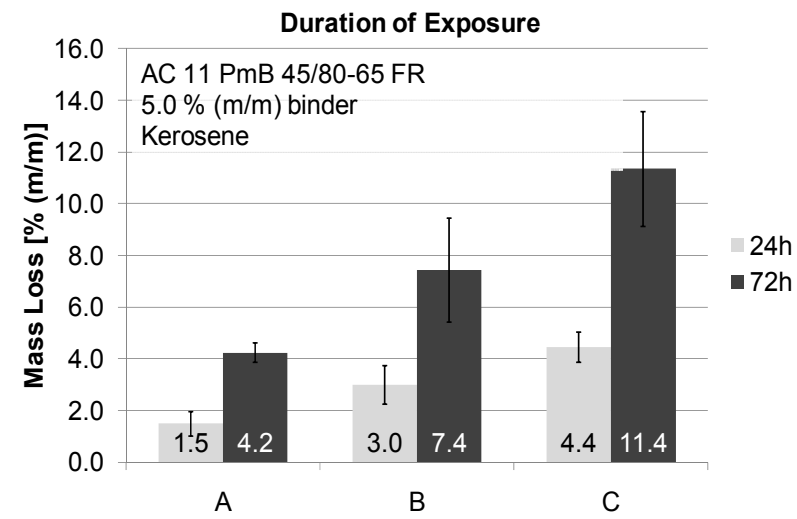

(a)

\subsection{Duration of Exposure}

To analyze the influence of the duration of exposure, specimens prepared by roller compaction (according to EN 12697-32) were tested with different immersion times. As an example, Fig. 5a gives the results for an AC $11 \mathrm{PmB}$ 45/80 FR and binder content of $5.0 \%(\mathrm{~m} / \mathrm{m})$. Four specimens were exposed for $24 \mathrm{~h}$, another four for $72 \mathrm{~h}$ in jet fuel (kerosene). As the volume of air voids is similar for all specimens $(M V: 4.7 \%(\mathrm{v} / \mathrm{v}), S D: 0.5 \%(\mathrm{v} / \mathrm{v}))$, the diagram shows $M V$ and $S D$ for the results of the two conditions.

The duration of exposure has a significant influence on the results. When the duration is increased by the factor of 3 from $24 \mathrm{~h}$ to $72 \mathrm{~h}$, the mass loss due to exposure increases by a factor of 2.8 and the mass loss due to abrasion by a factor of 2.5 .

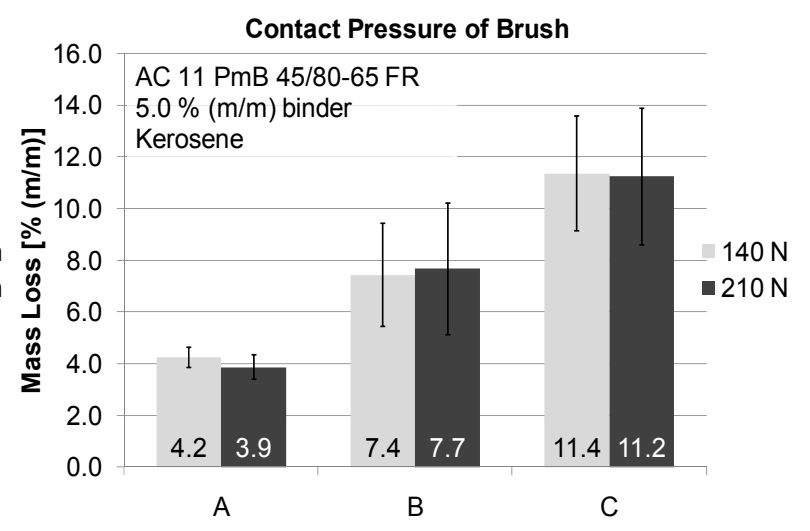

(b)

Fig. 5 Impact of (a) duration of exposure; (b)contact pressure of the brush. 


\subsection{Contact Pressure of Brush}

For the same mix as stated in Section 5.1, the contact pressure was varied while brushing. In one case, $140 \mathrm{~N}$ were used as required by the standard. In the other case, the weight was increased by $50 \%$ to 210 N. Fig. 5b gives information about the results. There is no significantly influence on the mass lost after abrasion (parameter $B$ ) due to a higher contact pressure. Thus it can be stated that the test seems to be insensitive to moderate changes in the contact pressure between brush and specimen. This might be explainable by the flexibility of the brush hair. A higher weight on the brush leads to greater deformation of the brush hair rather than a stronger abrasive force.

\subsection{Fuel Type}

Specimens of an AC $11 \mathrm{PmB} 45 / 80-65$ mix with $5.3 \%$ binder by mass were exposed to two different fuels (benzine and diesel) to find relevant influences of major fuel types. The specimens were exposed to the fuel for $72 \mathrm{~h}$ and then brushed acc. to $\mathrm{EN}$ 12697-43.

Three diagrams in Fig. 6 show parameters $A, B$ and $C$ vs. volume of air voids of the specimens including a linear regression to show dependencies on the volume of air voids. The upper-left diagram presents the results for mass loss after exposure (parameter $A$ ). Obviously, benzine shows the most aggressive behavior with more than $40 \%$ mass loss for the specimens at a higher volume of air voids. Diesel shows significantly lower aggressiveness. The volume of air voids has a crucial influence on mass loss due to exposure for benzine, less impact for diesel.

Parameter $B$, the mass loss due to abrasion is dependent on the volume of air voids to a much lower extent, but there is still an increasing trend. Exposure to benzine leads to lower resistance of the tested material to abrasive loading.

The combined parameter $C$ reflects the situation
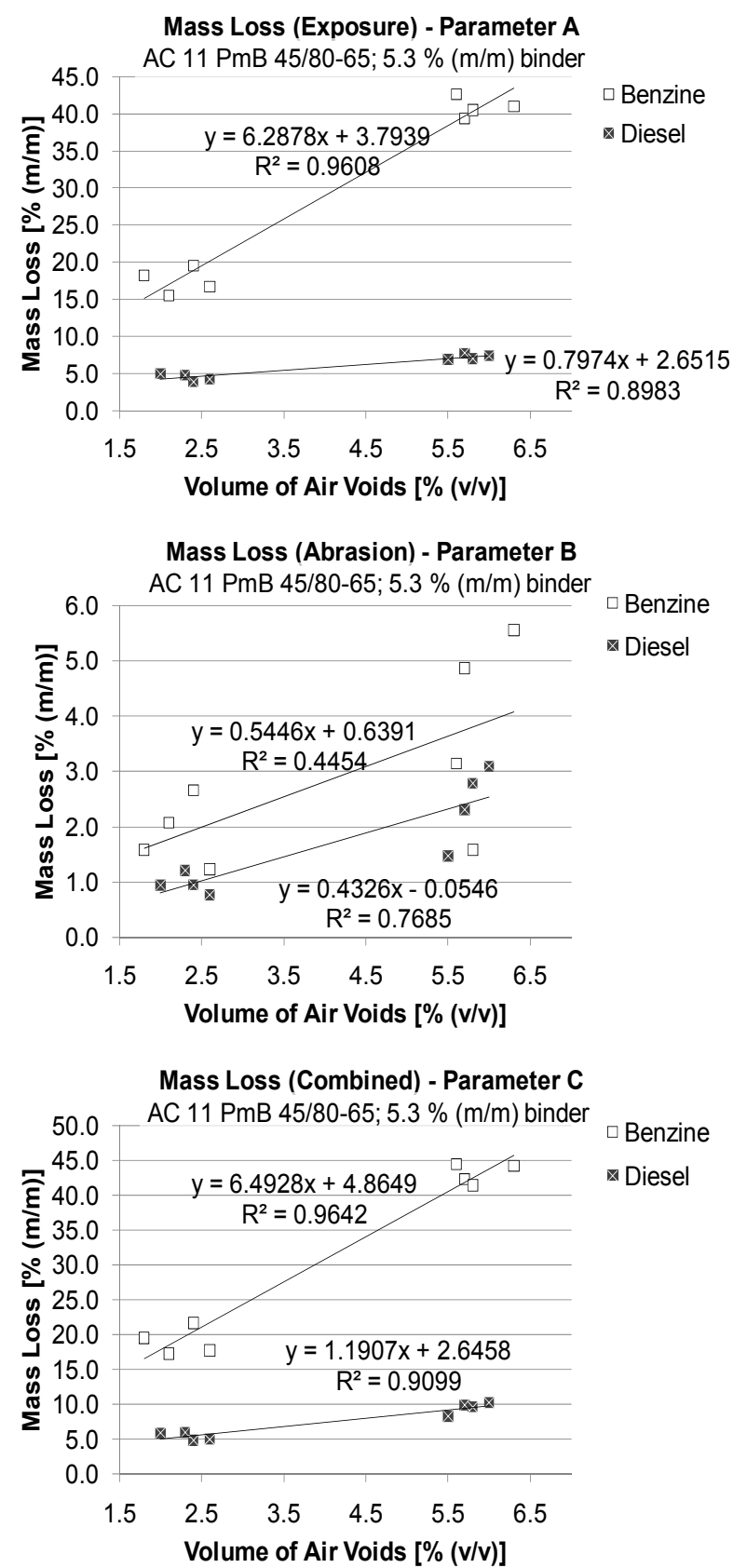

Fig. 6 Influence of fuel type on resistance.

found for parameter $A$ and $B$. Benzine is more aggressive showing a strong dependency on the volume of air voids. There is also an increasing trend for diesel but with a much lower slope.

\section{Conclusions}

From the research and the review of EN 12697-43, it is concluded that the standard is a sound basis for 
the assessment of fuel resistance of HMA with potential for improvement.

The suggestion is to implement the roller compactor into the standard EN 12697-33, especially because the roller compactor reproduces the compaction method used in road construction in the most reliable way.

It is recommended to set the height of the specimen produced in the laboratory in the standard more strictly (e.g. $63.5 \pm 2 \mathrm{~mm}$ ) or the immersed part of the specimen relative to its height (e.g. $50 \% \pm 1 \%$ ) to make sure that results from specimens with different heights can be compared.

A critical point is the cleaning of the specimen after exposure. The standard is imprecise in this point. As the research revealed, the method of washing and removing of loose particles significantly influences the results. Thus, the method of cleaning must be precisely given by the standard.

A new parameter $C$ was implemented that combines mass loss after exposure and brush testing. This parameter is independent of the method of cleaning. It is recommended to implement $C$ into $\mathrm{EN}$ 12697-43 and to obtain the fuel resistance according to parameter $C$ to ensure comparable and repeatable results.

A second example of a brush testing device should be given by the standard. The device constructed in the project is easy-to-handle, ensures that the requirements of the standards are met and produces comparable and repeatable results.

Regarding other influences of test conditions on the results, the duration of exposure has a significant impact as well as the type of fuel. Also, the volume of air voids has a clear impact on the test results. The weight pressing the brush to the specimen in the brush test was also varied but showed no significant change in the results.

\section{Acknowledgments}

The authors would like to express their gratitude for the funding of the research project by OMV Refining \& Marketing $\mathrm{GmbH}$.

\section{References}

[1] W.C. McBee, T.A. Sullivan, Improved resistance of sulfur-asphalt paving formulations to attack by fuels, Boulder City, USA, Industrial and Engineering Chemistry, Product Research and Development 16 (1) (1977) 93-95.

[2] F.G. Practico, R. Ammendola, R. Moro, Asphalt concretes for fuel-resistant roads, in: Proceedings of the 4th Eurasphalt \& Eurobitume Congress, Copenhagen, Denmark, 2008, pp. 995-1000.

[3] A. Seive, C. Leroux, F. Turquetil, Fuel Performance Evaluation of Asphalt Pavements, in: Proceedings of the 3rd Eurasphalt \& Eurobitume Congress, Vienna, Austria, 2004, pp. 191-202.

[4] D. Hanson, R. Boyer, G. King, AAPTP Project 05-02: Fuel Resistant Sealers and Binders for HMA Airfield Pavements, FAA (Federal Aviation Administration), 2009.

[5] ASTM D3910-Design, Testing and Construction of Slurry Seal, Annual Book of ASTM, USA, 1996.

[6] G.D. Airey, A.C. Collop, S. Zoorob, The Influence of Laboratory Compaction Methods on the Performance of Asphalt Mixtures, final report, Engineering and Physical Science Research Council, 2005.

[7] F.G. Pratico, R. Ammendola, A. Moro, Asphalt binder influence on chemical resistance of HMAs: A theoretical and experimental study, in: Proceedings of the 4th International SIIV Congress, Palermo, Italy, 2007. 